\title{
To Do or Not to Do: Whether to Hold or Continue Antithrombotics before Endoscopy
}

\author{
Joon Sung Kim and Byung-Wook Kim
}

Division of Gastroenterology, Department of Internal Medicine, Incheon St. Mary's Hospital, College of Medicine, The Catholic University of Korea, Seoul, Korea

See "Clinical Practice and Guidelines for Managing Antithrombotics before and after Endoscopy: A National Survey Study" by Seong Woo Jeon, et al. on page 316, Vol. 14, No. 3, 2020

Antithrombotics comprise antiplatelets (i.e., aspirin, clopidogrel) and anticoagulants (i.e., warfarin, apixaban). They are widely used for primary as well as secondary prevention of cardiovascular and cerebrovascular diseases. ${ }^{1}$ This translates to a large number of patients using antithrombotics before their endoscopic procedure. Physicians frequently consider the risks of continuing or stopping antithrombotics as well as the risks of thrombotic events or bleeding. Practice guidelines from the European Society of Gastrointestinal Endoscopy and the American Society of Gastrointestinal Endoscopy exist. ${ }^{2,3}$ Most recently, the Asian Pacific Association of Gastroenterology and the Asian Pacific Society for Digestive Endoscopy published a joint official statement regarding the management of antithrombotics undergoing endoscopy. ${ }^{4}$ These guidelines recommend management of antithrombotics based on two types of risks: bleeding risk and thrombotic risk. A diagnostic endoscopy with or without biopsy are considered procedures with low bleeding risk and cessation of antithrombotics are generally not required. A colonoscopy with polypectomy is a procedure with high bleeding risk. Management of antithrombotics depends on the type of antithrombotic (i.e., aspirin, warfarin) and the patient's risk of thrombosis. Endoscopic submucosa dissection (ESD) and endoscopic mucosal resection of polyps $\geq 2 \mathrm{~cm}$ are classified as ultrahigh risk procedures, and cessation of all antithrombotic is generally recommended.

In this issue of Gut and Liver, ${ }^{5}$ investigators from the Research Group for Endoscopic Submucosal Dissection in the Korean Society of Gastrointestinal Endoscopy performed a survey regarding the periprocedural management of antithrombotics for three distinct scenarios: forceps biopsy, colonic polypectomy, and gastric ESD. A total of 415 endoscopists completed the survey and nearly half (53\%) of the respondents were working in a general or tertiary hospital. The survey revealed that Korean doctors performed endoscopic biopsy without discontinuing antiplatelets but were reluctant to do so for anticoagulants. Most did not adhere to current guidelines and discontinued antiplatelets before polypectomy. Anticoagulants such as warfarin and apixaban were discontinued before polypectomy but heparin bridge therapy was erroneously considered in low-risk patients $(41.2 \%)$ and not considered in high-risk patients (35.2\%). The majority of doctors discontinued antithrombotics before gastric ESD. However, heparin bridge therapy was not selected in high thromboembolic risk patients in discordance with the current guidelines. The results of this survey report poor guideline adherence by a large number of Koreans, which is not surprising. Previous surveys from other countries have reported low compliance with the current guidelines. A survey from the United States reported that less than half of the endoscopy routinely continued aspirin before screening colonoscopy. ${ }^{6}$ Another survey from Japan reported that endoscopists were more likely to discontinue anticoagulants compared with antiplatelets. ${ }^{7}$

In this survey, the respondents were given a certain situation and were asked if they would perform the endoscopic procedure if the patient was taking antithrombotics. For example, the respondents were asked if they would perform a biopsy in patients with an active gastric ulcer if the patient was taking aspirin. This survey method probably overestimates the respondents' adherence to guidelines as they would generally respond

\footnotetext{
Correspondence to: Byung-Wook Kim

Division of Gastroenterology, Department of Internal Medicine, Incheon St. Mary's Hospital, College of Medicine, The Catholic University of Korea, 56 Dongsu-ro, Bupyeong-gu, Incheon 21431, Korea

Tel: +82-32-280-5052, Fax: +82-32-280-5987, E-mail: gastro@catholic.ac.kr

pISSN 1976-2283 eISSN 2005-1212 https://doi.org/10.5009/gnl20122

(9) This is an Open Access article distributed under the terms of the Creative Commons Attribution Non-Commercial License (http://creativecommons.org/licenses/by-nc/4.0) which permits unrestricted non-commercial use, distribution, and reproduction in any medium, provided the original work is properly cited.
} 
that they would perform a biopsy if a lesion was present. This does not correspond to recommending aspirin before their elective endoscopy.

The authors did not investigate the reasoning for the respondents' poor adherence to current guidelines. Poor guidelines adherence may stem from lack of familiarity or awareness of the guidelines especially regarding newer oral anticoagulants such as apixaban. They may also stem from distrust of the current guidelines as the evidence regarding the periendoscopic management of antithrombotics is generally of poor quality. It may also stem from the perceived risk of bleeding and their legal ramifications that follow such an event. Identifying the reasons should facilitate in narrowing the gap between the guidelines and clinical practices.

\section{CONFLICTS OF INTEREST}

No potential conflict of interest relevant to this article was reported.

\section{ORCID}

Joon Sung Kim Byung-Wook Kim https://orcid.org/0000-0001-9158-1012

https://orcid.org/0000-0002-2290-4954

\section{REFERENCES}

1. Abraham NS. Gastrointestinal bleeding in cardiac patients: epidemiology and evolving clinical paradigms. Curr Opin Gastroenterol 2014;30:609-614.

2. Gralnek IM, Dumonceau JM, Kuipers EJ, et al. Diagnosis and management of nonvariceal upper gastrointestinal hemorrhage: European Society of Gastrointestinal Endoscopy (ESGE) guideline. Endoscopy 2015;47:a1-a46.

3. ASGE Standards of Practice Committee, Acosta RD, Abraham NS, et al. The management of antithrombotic agents for patients undergoing GI endoscopy. Gastrointest Endosc 2016;83:3-16.

4. Chan FKL, Goh KL, Reddy N, et al. Management of patients on antithrombotic agents undergoing emergency and elective endoscopy: joint Asian Pacific Association of Gastroenterology (APAGE) and Asian Pacific Society for Digestive Endoscopy (APSDE) practice guidelines. Gut 2018;67:405-417.

5. Jeon SW, Hong SJ, Lee ST, Kim H, Chun HJ; Research Group for Endoscopic Submucosal Dissection in Korean Society of Gastrointestinal Endoscopy. Clinical practice and guidelines for managing antithrombotics before and after endoscopy: a national survey study. Gut Liver 2020;14:316-322.

6. Robbins R, Tian C, Singal A, Agrawal D. Periprocedural management of aspirin during colonoscopy: a survey of practice patterns in the United States. Gastrointest Endosc 2015;82:895-900.

7. Tsuruoka N, Iwakiri R, Sakata Y, et al. Questionnaire-based survey on gastrointestinal bleeding and management of antithrombotic agents during endoscopy among Asian countries. Digestion 2018; 97:97-106. 